\title{
Profiling the differentially expressed genes in two rice varieties during rapid grain- filling stages
}

\begin{abstract}
Grain filling is an important agronomic trait, which directly affects the final yield of rice. Partially filled and empty rice grains are among the factors that limit the yield of MR219, one of the highest yielding rice varieties in Malaysia. In this study, the NSF $20 \mathrm{~K}$ rice oligonucleotide array, which contains 20,000 70-mer oligonucleotide probes, was used for direct comparison of the transcriptomes of MR219 and MR84 (a rice variety that has higher percentage of filled grains compared to MR219), during rapid grain-filling period at 5 and 10 days after fertilization (DAF). A total of 155 and 233 genes were differentially expressed in MR219 compared to MR84 at 5 and 10 DAF, respectively; and 9 of these expression ratios were tested using quantitative real-time RT PCR. Among the differentially expressed genes identified were those encoding hexokinase, various sugar transporters, GSDL-like lipase/acylhydrolase, brassinosteroid-insensitive 1-associated receptor kinase 1 precursor and homeobox protein GLABRA2, which were analyzed by real-time RT PCR in this study. The differences demonstrated by these genes in their transcript levels and profiles, between the two rice varieties understudied at different stages of grain filling may contribute to the formulation of hypotheses toward the understanding of poor percentage of filled grains in MR219.
\end{abstract}

Keyword: Grain filling; Oligonucleotide array; Rice; Sugar signaling Transportation of assimilates 